REVISTA

MEXICANA DE

ECONOMÍA Y

FINANZAS

REMEF

(THE MEXICAN JOLRNAL OF

ECONOMICS AND FINANCE)
Revista Mexicana de Economía y Finanzas, Nueva Época

Volumen 16 Número 3, Julio - Septiembre 2021, pp. 1-18, e652

THE ECONOMICS AND FINANCE EFFECTS OF THE COVID-19 PANDEMIC Editor: Dr. Ignacio Perrotini

DOI: https://doi.org/10.21919/remef.v16i3.652

(Recibido: 30/marzo/2021, aceptado: 2/julio/2021, publicado: 2/julio/2021)

\title{
Dependencia de los mercados de valores de Argentina, Brasil y México respecto del estadounidense: Covid19 y otras crisis financieras recientes
}

\author{
Domingo Rodríguez Benavides ${ }^{1}$ - Universidad Autónoma Metropolitana, México \\ César Gurrola Ríos - Universidad Juárez del Estado de Durango, México \\ Francisco López Herrera - Universidad Nacional Autónoma de México, México
}

Este artículo muestra el análisis efectuado para comparar la estructura de dependencia de la caída de los rendimientos accionarios de los mercados de los tres países más grandes de América Latina (Argentina, Brasil y México) en los rendimientos del mercado de valores estadounidense. Se compara la caída propiciada por el surgimiento de la pandemia de Covid-19 con otros episodios de crisis financieras acaecidas a partir de la última década del siglo XX. Mediante cópulas bivariadas de Clayton con regímenes conducidos por cambios markovianos, se estudia el grado de la dependencia en la cola inferior de la distribución, encontrando patrones distintos de dependencia que sugieren que los mercados de Brasil y México son más dependientes que el argentino. Una limitante es que no termina aún la recuperación económica derivada de la pandemia, por lo que los resultados en relación con los efectos de la Covid-19 podrían considerarse sólo como provisionales, sin embargo, este artículo contribuye a entender cómo ha sido el proceso de globalización y las posibles consecuencias para la internacionalización de los mercados bursátiles latinoamericanos y la inversión en la región, incluyendo la de portafolio.

Clasificación JEL: F36, F65, G11, G12, G15.

Palabras clave: Covid-19, mercados accionarios, caídas bursátiles, cópula Clayton, cambios markovianos

\section{Dependence of the stock markets of Argentina, Brazil and Mexico with respect to the U.S.: Covid19 and other recent financial crises}

This paper compares the dependency structure of the fall of equity returns in the three largest Latin American countries'stock markets. The fall caused by the emergence of the Covid-19 pandemic is compared with other episodes of financial crises that occurred since the last decade of the 20th century. Using bivariate Clayton copulas with regimes driven by Markovian changes, the degree of dependence in the lower tail of the distribution is studied, finding different patterns of dependence that suggest that the markets of Brazil and Mexico are more dependent than the Argentinean. A limitation is that the economic recovery derived from the pandemic has not yet ended, so the results in relation to the effects of Covid-19 could only be considered provisional. However, this article contributes to understanding the process of globalization and the possible consequences for the Latin American stock markets' internationalization and the investment in the region, including portfolio investment.

JEL Classification: F36, F65, G11, G12, G15.

Keywords: Covid-19, stock markets, stock market crashes, Clayton copula, markovian switching.

1 Autor de correspondencia. Universidad Autónoma Metropolitana (UAM) Azcapotzalco

Email: dorobe@azc.uam.mx

*Sin fuente de financiamiento para el desarrollo de la investigación 


\section{Introducción}

Con el objetivo de comparar la relación entre los rendimientos de los mercados accionarios de las tres principales economías latinoamericanas respecto del mercado estadounidense, se estiman cópulas bivariadas con resultados que muestran las diferencias y similitudes entre la caída atribuible a la pandemia de Covid-19 y las caídas bursátiles asociadas con las crisis financieras de la última década del siglo pasado y lo que va del presente siglo. Se observaron similitudes entre el cambio del grado de dependencia de los mercados latinoamericanos respecto del estadunidense, aunque también notables diferencias que sugieren que, a pesar de que ha aumentado la integración financiera entre esos mercados, pueden existir oportunidades de diversificación de portafolios.

Casi dos décadas después del brote del Síndrome Respiratorio Agudo Severo (SARS), Asia enfrentó una variante epidémica por Coronavirus que ocasiona la enfermedad denominada COVID19. Esta nueva epidemia propició, como lo señalan Ftiti, Ben Ameur y Louhichi (2021) entre otros, efectos en los sistemas y mercados financieros alrededor del mundo debido a las noticias relacionadas con esa epidemia. Los datos de la Organización Mundial de la Salud muestran que a fines del 2020, se habían infectado con COVID-19 en todo el mundo 82.4 millones de personas, causando alrededor de 1.8 millones de muertes. Al-Awadhi et al., (2020) encuentran que el incremento de los contagios afectó negativamente a los rendimientos bursátiles. Además de las pérdidas humanas y grandes costos erogados por los sistemas de salud, las economías de todo el orbe se han visto severamente afectadas, causando una erosión en el valor de los mercados bursátiles globales del orden del 30\% durante los primeros meses del periodo COVID-19 según Ali, Alam y Rizvi (2020). El fuerte daño económico obedeció al alto grado de contagio del COVID-19 así como a los confinamientos ordenados por los gobiernos de todo el mundo.

Los desafíos económicos causados por la pandemia y el simultáneo colapso del precio del petróleo no son el primer choque que los mercados emergentes enfrentan. Por ejemplo, en 1994 México experimentó la crisis denominada "efecto tequila", Brasil sufrió la crisis del "efecto samba" en 1999 y posteriormente Argentina padeció los efectos de la "crisis del corralito". Además, en las últimas décadas, el creciente flujo de inversiones y comercio transfronterizo en una economía mundial cada vez más entrelazada ha sido objeto de repetidas interrupciones. No obstante, en todas las crisis hay determinadas condiciones económicas que pueden contribuir a profundizar o a agravar las crisis financieras ${ }^{2}$.

Poco después del inicio de la pandemia de Covid-19, los mercados emergentes observaron un colapso de la demanda interna y externa, salidas de capital, aumento de los costos de los préstamos externos, caída de precios de las materias primas y depreciación de las monedas. Los gobiernos de esos países emergentes, al igual que los de las economías avanzadas, aumentaron el endeudamiento interno mediante políticas no convencionales para reunir recursos fiscales para luchar contra la

\footnotetext{
2 Para una revisión de las condiciones económicas que gestaron las anteriores crisis se recomienda remitirse, a sugerencia de uno de los dictaminadores, en el caso de Argentina a: Damill, Frenkel y Rapetti (2015); para Brasil: Carvalho (2018); y en el caso de México: de Souza y Gómez-Ramírez (2018), Moreno-Brid y Ross (2009) y Moreno-Brid y Ross (2010). Desde luego una amplia revisión y discusión sobre estos temas escapa a los fines de nuestro trabajo.
} 
pandemia. Además, la desaceleración general del crecimiento de los mercados emergentes comenzó en 2013 con el taper tantrum ${ }^{3}$ de la Reserva Federal (Fed).

Las crisis financieras de los mercados emergentes han tenido diferentes causas externas e internas, siendo la producida por Covid-19 de naturaleza particular pues su principal detonador es una crisis sanitaria. El análisis de Daehler, Aizenman y Jinjarak (2021) muestra que antes de la crisis por Covid-19 se encontraban tanto factores mundiales como regionales entre los principales causales del riesgo financiero de los mercados emergentes, durante la irrupción de la pandemia los principales factores de riesgo resultaron ser el espacio fiscal y la dependencia del petróleo. Estas diferencias en esos factores de riesgo se pueden explicar, al menos en parte, por el margen de los países emergentes para enfrentar la contingencia y continuar con sus políticas públicas y proyectos durante ella; así como por la guerra de precios petroleros presente a principios de 2020.

Las principales crisis financieras ocurridas a fines del siglo XX surgieron de los mercados emergentes, contaminando en algunos casos a los mercados desarrollados o maduros. Por lo contrario, las primeras crisis del siglo XXI se originaron en los mercados estadounidenses y europeos. La crisis financiera producto de la pandemia de Covid-19, como argumentan Cakmaklı, Demiralp, Kalemli-Ozcan, Yesiltas y Yıldırım (2020), puede desencadenar una gran crisis de los mercados emergentes con posibles efectos de contagio hacia los países avanzados. Por tal motivo, sigue siendo de importancia estudiar las relaciones entre los mercados de capital de los países emergentes en relación con los desarrollados. Este artículo contribuye al estudio de esas relaciones, analizando cómo se han vinculado las caída de los rendimientos de los mercados accionarios argentino, brasileño y mexicana con respecto de las caídas en los rendimientos de la bolsa estadunidense.

El resto del documento tiene la siguiente estructura. La siguiente sección presenta una revisión de literatura relevante, seguida por la descripción del aparato metodológico empleado. Posteriormente se muestran los resultados del análisis de los datos y la última sección enuncia las conclusiones.

\section{Revisión de literatura}

El antecedente moderno de la integración de los mercados de capitales, como una extensión de la globalización económica y financiera, se podría asociar al desplome de la actividad económica global consecuencia de ajustes económico-financieros posteriores a la Segunda Guerra Mundial del siglo $\mathrm{XX}^{4}$. Como respuesta a tales desafíos se propusieron e implementaron, desde economías desarrolladas encabezadas por Estados Unidos e Inglaterra, profundas reformas económicas y financieras a favor del libre mercado y en contra de iniciativas regulatorias sobre el flujo de las inversiones. De esta manera, los mercados de capital domésticos permitieron la libre movilidad de capitales desde y hacia el exterior, no sólo en los países desarrollados sino también, y cada vez con mayor intensidad, en sus contrapartes emergentes (Vargas y Bayardo, 2013; López y Ortiz, 2010; López et al, 2019).

\footnotetext{
3 Término que se acuñó para definir la reacción de los mercados a la reducción de las políticas expansivas llevadas a cabo por la FED.

${ }^{4}$ La creación y auge del euromercado, el rompimiento de los acuerdos de Bretton Woods, la caída de los precios del petróleo de los años 70's, la crisis de deuda de países emergentes, entre otros.
} 
La eliminación de barreras al flujo de capital, particularmente el destinado hacia inversiones en activos financieros -portafolios-, así como una mayor sofisticación y sincronización tecnológica5, por un lado, y la creación de novedosos instrumentos financieros que pretenden mejorar las posibilidades de inversión, financiamiento, cobertura, arbitraje por otro ${ }^{6}$, han logrado, entre otros aspectos, un notable incremento en el nivel de integración entre los mercados financieros del mundo (Dorodnykh, 2014; Sandoval et al. (2015); Mellado y Escobari, 2015; Campos y Revoredo, 2017; Kumamoto y Zhuo, 2020).

La transmisión de efectos entre distintas economías y mercados es otra explicación del incremento notable en los estudios sobre integración financiera; entre los esfuerzos pioneros que discuten la propagación de efectos a través de los mercados, en el ámbito de las crisis que se gestaron desde la década de los 1990, figuran Calvo y Reinhart (1996), Rigobón (2002), Forbes y Rigobon (2002): Beaker y Harvey (2003), Bekaert et al. (2005) y Bucio, Gutiérrez, y Sosa (2017). La crisis de mercados emergentes de los 19907, la crisis hipotecaria del mercado de EU del 2007 y la crisis de deuda europea de 2010, son ejemplos recientes de descalabros financieros relevantes, en función del impacto negativo de tales shocks en los mercados internacionales (Santillán, Gurrola y López et al., 2017).

En la medida en que se han venido fortaleciendo los vínculos en los mercados financieros los efectos de las perturbaciones de corto plazo en uno de ellos se transmiten, en función del nivel de integración, a los otros e incluso se ha observado que en el largo plazo comparten tendencias comunes (Kasa, 1992). Antes de la década de los noventa eran escasos los estudios que se interesaban por explicar la forma en que los mercados emergentes reaccionaban a la dinámica financiera de otras economías; la crisis de México en 1994, mejor conocida como "efecto tequila", detonó el interés por su estudio (Calvo y Reinhart, 1996). Entre los esfuerzos pioneros que abordan la discusión sobre la propagación de efectos entre mercados financieros, en especial en el ámbito de las crisis financieras que se gestaron desde la década de los 90’s , figuran Calvo y Reinhart (1996), Rigobón (2002), Forbes y Rigobon (2002). En tales trabajos, así como en aquellos que siguieron su enfoque, se popularizó el término "contagio" para hacer referencia a la citada transmisión de efectos económicos y financieros. La posibilidad de transmisión de efectos entre mercados tiene implicaciones importantes sobre las decisiones de inversión internacional, particularmente sobre el precio de los activos y el consecuente interés de formar portafolios que permitan diversificar el riesgo sistemático de los mercados domésticos (López y Venegas, 2012).

No obstante, la creciente popularidad del término "contagio", es importante considerar tres aspectos fundamentales. En primer lugar, el riesgo de sobrevalorar las relaciones provenientes de la dinámica normal entre los mercados a causa de la sensibilidad a la volatilidad de los datos, así como a causa de las propiedades estadísticas de las series financieras -heterocedasticidad, autocorrelación

\footnotetext{
${ }^{5}$ Los avances tecnológicos detonaron, además de la rapidez en las transacciones, el desvanecimiento de las distancias entre los mercados; de esta manera, al mismo tiempo que la oferta de opciones para los residentes de una economía se ha incrementado las operaciones son más frecuentes y casi instantáneas (Márquez et al., 2003).

${ }^{6}$ Lo que a su vez implica mejorar las posibilidades de diversificación, la reducción del costo de capital y el aumento en el valor de las empresas.

7 Calvo y Reinhardt (1996), Rigobon (2002), Mendoza, López y Watkins (2011) y Santillán (2015) ofrecen una amplia discusión sobre los detalles de las crisis financieras de finales del siglo XX.
} 
y ausencia de normalidad (Forbes y Rigobon, 2002). Por otro lado, la evidencia empírica disponible sugiere que se pueden identificar efectos aleatorios que acompañan a los shocks en los mercados finanacieros que son completamente normales y que, en ocasiones, tienden a confundirse con algún tipo de contagio. En tercer lugar, en lugar de atribuir la mayor parte del contagio a las relaciones comerciales entre los mercados involucrados, hay que reconocer la posibilidad de que la aparente transmisión sólo es el resultado de que un determinado grupo de activos coticen simultáneamente en tales mercados (Rigobon, 2002; Calvo y Reinhart, 1996; Hernández y Valdés, 2001).

Aunque los esfuerzos por fortalecer los vínculos comerciales y financieros, promover el libre flujo de capitales, eliminar restricciones regulatorias, entre otros aspectos, han logrado en cierta medida, mejorar el desempeño de mercados desarrollados su efecto sigue siendo objeto de estudio en sus contrapartes emergentes ${ }^{8}$. Diversos estudios han documentado en la región de América Latina que el fortalecimiento del comercio exterior contribuye espacios de integración financiera (Rigobon, 2002; Frenkel, 2003; Mollah y Hartman, 2012; Marcet, 2017); no obstante, también existen estudios que aseguran que la reducción en los costos de transacción y en el costo de capital, pueden entorpecer las decisiones de inversión del capital internacional en su intento por reducir los niveles de exposición al riesgo sistemático doméstico, vía la diversificación de portafolio (Romero et al, 2013; Sandoval y Soto, 2016; Mellado y Escobari, 2015). Por otro lado, la evidencia empírica disponible sugiere que los beneficios de la integración financiera no se aprecian, necesariamente, en forma homogénea entre los mercados participantes (Espinosa-Méndez, Gorigoitía y Vieito; 2017); adicionalmente existen ciertos aspectos que pueden interferir con una integración exitosa como la armonización financiera, el tamaño de los mercados domésticos y aspectos regulatorios (Dorodnykh, 2014).

Múltiples estudios muestran evidencia empírica sobre la influencia que los mercados de capitales de la economía estadounidense tienen sobre la dinámica bursátil y económica mundial, en especial la que se desarrolla en mercados menos desarrollados. Por ejemplo, la transmisión de efectos financieros de la crisis subprime se ha documentado por Narayan et al. (2014) al comprobar el incremento en las correlaciones condicionales en India, Australia y China; Nguyen et al. (2017) comprueban contagio entre mercados de Vietnam y China versus el mercado de Estados Unidos, antes y después de la crisis; Bucio et al., (2017) demuestran el efecto contagio a causa de la crisis subprime en los países que pertenecen al TMEC mediante la modelación de cópulas dinámicas. Mohti et al. (2019) comprueba efectos diferenciados sobre dieciocho mercados bursátiles en países de América Latina, Europa, África, Oriente Medio y Asia, tomando como referencia las crisis subprime y la de deuda en la eurozona.

Otros estudios sobre las relaciones entre mercados de valores en Asia son Abid, Kaabia y Guesmi (2014) quienes encuentran que el mercado bursátil de EU afecta, en el tiempo y entre los mercados, el proceso de integración; Valadkhani y Chancharat (2008) constatan posibilidades de diversificación de portafolios en la región al rechazar la existencia de relaciones de largo plazo, mientras que Veerappa (2016) documenta relaciones de corto y largo plazo. Chernov, Creal y Hördahl

\footnotetext{
${ }^{8}$ Los que han adoptado tales estrategias con la finalidad de atraer inversión extranjera que permita, entre otras cosas, corregir desequilibrios financieros internos y promover el crecimiento. Por supuesto que para lograrlo estos países se ven obligados a ofrecer, por un lado, atractivos rendimientos que compensen a los inversionistas extranjeros por los riesgos domésticos y por otro, importantes incentivos fiscales y ventajas comparativas en los procesos productivos.
} 
(2020) y Kumamoto y Zhuo, J. (2020) documentan la influencia del mercado de EU sobre la dinámica de los bonos soberanos en Asia.

En Europa diversos estudios comprueban relaciones de cointegración y crecientes correlaciones condicionales dinámicas tomando como referencia el mercado de EU (Bartra y Wang, 2007; Gjika y Horvath, 2013; Boubaker y Jouini, 2014; Munteanu et al. 2014; Trabelsi y Hmida (2018); Fry-McKibbin et al. 2021). En los mercados BRIC Kenourgios et al. (2011) también reportan el efecto contagio. En los mismos mercados más Sudáfrica, Mensi et al. (2016) demuestran integración fraccional en la volatilidad persistente de los rendimientos y correlaciones dinámicas con el mercado de EU. Shahateet (2019) encuentra relaciones débiles en los rendimientos de los mercados de valores árabes. Carrieri et al. (2007) documentan una tendencia creciente en la integración financiera, aunque en distintos grados, en ocho países emergentes siendo México el más integrado y la India el más segmentado. López et al. (2009) ofrecen evidencia de integración en el TMEC. Mediante la metodología wavelet Dima et al. (2015) demuestra integración y procesos de sincronización de corto y mediano plazo en los mercados de México, Brasil y Chile. Más recientemente, López et al. (2019) señala la necesidad de reconocer en la dinámica de los principales mercados latinoamericanos shocks o perturbaciones exógenos entre los que destaca el comportamiento de los mercados de capital en EUA.

Akhtaruzzaman, Boubaker y Sensoy (2021) investigan el contagio financiero durante la crisis del COVID-19 y encuentran que las firmas financieras juegan un papel prominente en la transmisión de contagio en comparación con las firmas no financieras. Ali et al. (2020) establecen las conexiones entre la propagación del COVID-19 y los mercados financieros globales. Observaron una caída pronunciada en los mercados financieros globales en tanto que el mercado chino se estabilizó en un tiempo relativamente corto a partir de la crisis sanitaria.

Corbet, Hou, Hu y Oxley (2020) prueban la presencia de spillovers de volatilidad de los mercados financieros chinos durante el brote de la pandemia de COVID-19 en una amplia número de activos financieros tradicionales, sus resultados indican impactos excepcionalmente pronunciados y persistentes del coronavirus en los mercados financieros chinos en comparación con el índice de influenza tradicional. Löwen, Kchouri y Lehnert (2021) que la crisis financiara originada por la pandemia comparte rasgos semejantes a las de otras crisis, particularmente siendo de importancia los efectos entre diferentes mercados producios por la búsqueda de activos más seguros. Sin embargo, Cound (2021) sugiere que es importante saber diferenciar entre riesgo de mercado (como lo que se estudia en este artículo) y riesgo sistémico. Por otra parte, Grable (2021) analiza 15 episodios críticos caracterizados por fuertes correcciones y pánicos ocurridos durante 200 años de funcionamiento de la bolsa neoyorkina, argumentando que la volatilidad asociada a la reacción inicial de ese mercado a la pandemia por Covid-19 no se puede considerar como un evento extremo ni como una situación no esperada. 


\section{Metodología}

A partir del estudio pionero de Embrechts et al. (1999), las cópulas se han utilizado ampliamente para estudiar diversas relaciones en el ámbito financiero. Una cópula es una función con $d$ dimensiones

$$
F\left(x_{1}, \ldots, x_{d}\right)=C\left(F_{1}\left(x_{1}\right), \ldots, F_{d}\left(x_{d}\right)\right)
$$

Si todas las funciones de distribución marginal son continuas y si la función cópula es siempre creciente en las variables uniformes (posiblemente generadas a partir de una transformación) involucradas y si, además, la distribución conjunta es también creciente, entonces la cópula es única y cualquier función $C:[0,1]^{d} \rightarrow[0,1]$ implica una cópula. Las cópulas con dos dimensiones son relativamente las más sencillas y es suficiente para el caso que nos ocupa, por tal motivo en lo sucesivo nos ocuparemos de ellas, sin pérdida de generalidad pues los conceptos respectivos se pueden extender fácilmente al contexto de las cópulas multivariadas.

Si $X$ y $Y$ son dos variables aleatorias, en este trabajo los rendimientos del mercado accionario estadounidense, $X$, y los rendimientos de uno de los mercados analizados ( $Y=$ Argentina, Brasil o México), con distribución conjunta $F_{X, Y}(x, y)$ y cuyas respectivas funciones de distribución marginal son $F_{X}(x)$ y $F_{Y}(y)$, entonces, de acuerdo con el Teorema de Sklar (1959), existe una descomposición única de dicha distribución conjunta en las funciones de distribución marginal

$$
F_{X, Y}(x, y)=C\left(F_{X}(x), F_{Y}(y)\right)
$$

y en la cópula

$$
C\left(u_{1}, u_{2}\right)=P\left(U_{1} \leq u_{1}, U_{2} \leq u_{2}\right), U_{1} \equiv F_{X}(x), U_{2} \equiv F_{Y}(y)
$$

definida en $[0,1] \times[0,1]$, se encuentra contenida toda la información acerca de la estructura subyacente de la dependencia entre las variables $X$ e $Y$.

En casos como el de la distribución normal o de la $t$ de Student, ambas miembros de la familia de distribuciones elípticas, el grado de la dependencia entre dos variables se puede medir de manera relativamente sencilla por medio del llamado coeficiente de correlación de Pearson. Sin embargo, para otros tipos de distribuciones se puede requerir una medida alternativa para describir el vínculo existente entre dos variables. Según Nelsen (2006), dos pares de variables aleatorias $(X, Y),(\bar{X}, \bar{Y})$ son concordantes cuando $X<Y \Rightarrow \bar{X}<\bar{Y}$ o $X>Y \Rightarrow \bar{X}>\bar{Y}$, y son discordantes si $X<Y \Rightarrow \bar{X}>\bar{Y}$ o $X>Y \Rightarrow \bar{X}<\bar{Y}$; a partir de lo cual se puede definir la tau de Kendall como la diferencia entre la probabilidad de concordancia y la probabilidad de discordancia (no covariación):

$$
\tau[X, Y] \equiv P[(X-\tilde{X})(Y-\tilde{Y})>0]-P[(X-\tilde{X})(Y-\tilde{Y})<0]
$$

De acuerdo con Schweizer y Wolff (1981), la tau de Kendall se puede definir como 


$$
\tau(X, Y) \equiv 4 \iint_{[0,1]^{2}} C\left(u_{1}, u_{2}\right) d C\left(u_{1}, u_{2}\right)-1=4 E\left[C\left(u_{1}, u_{2}\right)\right]-1
$$

Como un hecho bastante conocido tenemos que por lo regular las distribuciones de los rendimientos financieros se alejan de un comportamiento similar a la distribución normal, particularmente en forma de sesgos respecto de las medias y alta curtosis, produciendo observaciones en las colas de la distribución con mayor probabilidad de ocurrencia de lo que cabría esperar en el caso de una variable que sigue una distribución normal. En el caso del movimiento conjunto de mercados, las regiones de las colas de la distribución son de gran interés por la probabilidad asociada con la ocurrencia de eventos extremos en un mercado y sus efectos en otro(s) mercado(s); relación que se analiza al tomar en cuenta la llamada dependencia de la cola. La aplicación de la metodología de cópulas permite estudiar tanto las colas gordas como el grado de la dependencia existente en ellas, el punto de partida es considerar la concordancia entre los valores extremos de la distribución conjunta, es decir, la posibilidad de que ocurra un evento con probabilidad menor que $\pi$ en la variable $X$, dado que en la variable $Y$ ocurre un evento con probabilidad menor que $\pi$ :

$$
\lambda(\pi) \equiv P(X \leq \pi \mid Y \leq \pi)=\frac{P(X \leq \pi, Y \leq \pi)}{P(Y \leq \pi)}=\frac{C(\pi, \pi)}{\pi} .
$$

A partir de esto se puede obtener una estimación del grado de dependencia tanto en la cola inferior como en la cola superior, mediante los respectivos índices de cola:

$$
\begin{gathered}
\lambda_{I} \equiv \lim _{\pi \rightarrow 0^{+}+} \frac{C(\pi, \pi)}{\pi}, \\
\lambda_{S} \equiv \lim _{\pi \rightarrow 1^{-}-} \lambda_{\pi} \equiv \lim _{\pi \rightarrow 1^{-}-} \frac{P(\bar{X}>\pi, \bar{Y}>\pi)}{P(\bar{Y}>\pi)}=\lim _{\pi \rightarrow 1^{-}-} \frac{1-2 \pi+C(\pi, \pi)}{1-\pi} .
\end{gathered}
$$

Entre las diferentes familias de cópulas, la gran variedad que forma parte de la familia arquimediana ha ganado popularidad debido a la facilidad con que se construyen, algunas de esas cópulas incluso requieren sólo un parámetro que gobierna la fuerza de la dependencia, y también se han popularizado porque por medio de ellas se puede modelar diferentes tipos de dependencia. Además, las cópulas de esta familia no se derivan de funciones de distribución multivariadas y la mayoría tiene expresiones en forma cerrada. Siguiendo a Nelsen (2006), si $\varphi$ es una función continua, estrictamente decreciente de $[0,1]$ a $[0, \infty]$ tal que $\varphi(1)=0$ y si $\varphi^{-1}$ es su inversa; entonces la función de $[0,1]^{2}$ a $[0,1]$ dada por

$$
C\left(u_{1}, u_{2}\right)=\varphi^{-1}\left(\varphi\left(u_{1}\right)+\varphi\left(u_{2}\right)\right)
$$

es una cópula si y sólo si es convexa $\varphi$, conocida como la función generadora de la cópula. 
Si $\varphi^{-1}$ se puede diferenciar dos veces, entonces la densidad de la cópula es

$$
c\left(u_{1}, u_{2}\right)=\frac{\varphi^{-1^{\prime \prime}}\left(\varphi\left(u_{1}\right)+\varphi\left(u_{2}\right)\right)}{\varphi^{-1^{\prime}}\left(\varphi^{\prime}\left(u_{1}\right)\right) \varphi^{-1^{\prime}}\left(\varphi^{\prime}\left(u_{2}\right)\right)},
$$

y la tau de Kendall está dada en este caso por

$$
\tau(C)=1+4 \int_{0}^{1} \frac{\varphi(t)}{\varphi^{\prime}(t)} d t
$$

La copula bivariada Clayton está dada por:

$$
C_{\theta}^{C L}\left(u_{1}, u_{2}\right)=\left(u_{\{1}{ }^{-\theta}+u_{\{2}\right\}^{-\theta}+1^{-\frac{1}{\theta}}
$$

Donde $\theta \in[1, \infty) \backslash\{0\}$. Cuando $\theta \rightarrow 0$ describe la independencia de la copula, mientras que para $\theta \rightarrow \infty$ describe la comonotonicidad de la copula.

Las cópulas son muy útiles en el estudio de la dependencia debido a sus propiedades. En primer lugar, son invariantes a las transformaciones estrictamente crecientes de las variables aleatorias. En segundo lugar, calculan medidas de concordancia consistentes, como la tau de Kendall utilizada en este estudio para indicar el grado de dependencia. En tercer lugar, también se presenta un tratamiento relevante de la dependencia asintótica de la cola.

\section{Análisis de los rendimientos accionarios}

Los datos diarios de los índices bursátiles de Argentina (Merval), Brasil (Bovespa), Estados Unidos (S\&P 500) y México (IPC) se obtuvieron de yahoo finance y cubren del 27 de abril de 1993 al 26 de marzo de 2021.
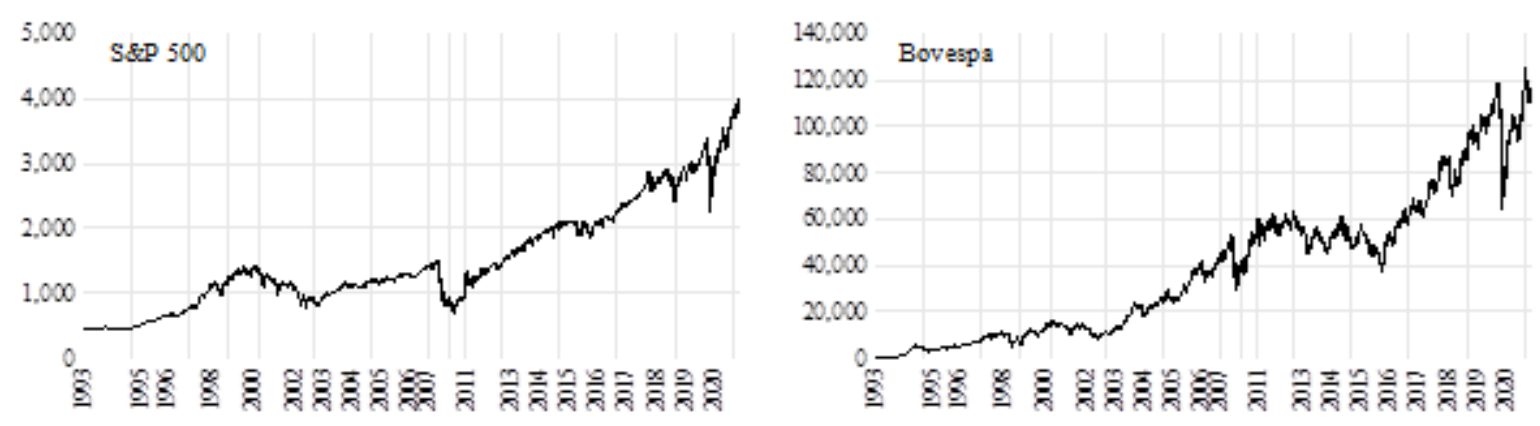

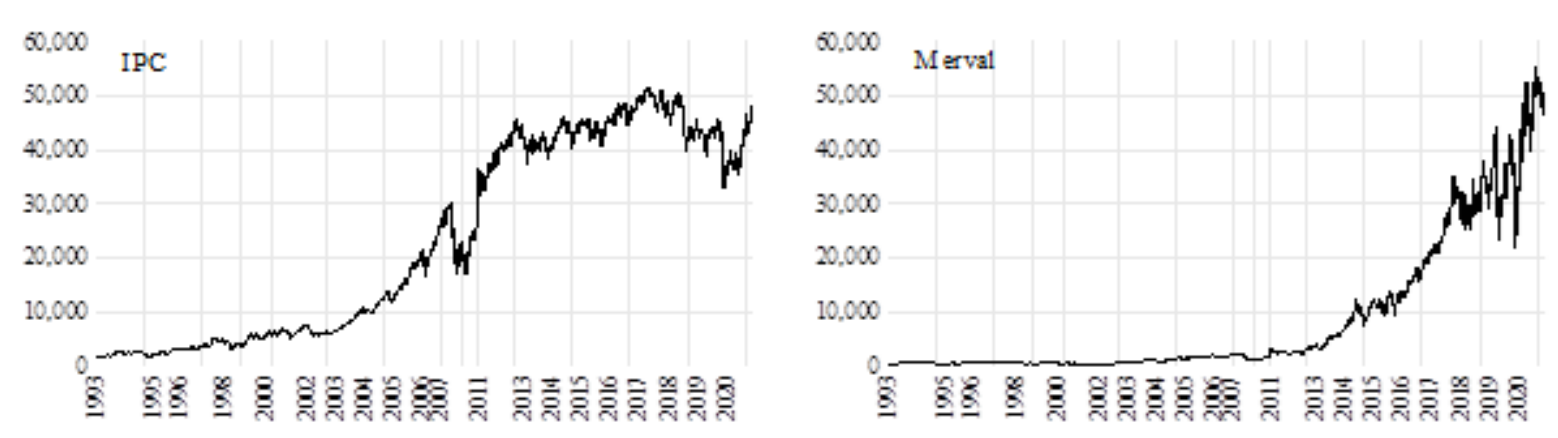

Figura 1. Índices de los cuatro mercados bursátiles

En la figura 1 se puede ver que los cuatro índices bursátiles han tenido una tendencia similar creciente, incluso recuperándose de manera similar para casi todas las crisis financieras ocurridas durante el periodo analizado, aunque es de destacarse que el indicador mexicano muestra un estancamiento caracterizado por una tendencia descendente desde el segundo semestre de 2017. Aunque en la perspectiva general ese estancamiento del mercado mexicano hace que su comportamiento sea distinto al de los otros tres mercados, se ha recuperado una parte importante de la caída producida durante el surgimiento de la Covid-19 y continúa ascendiendo hasta superar los niveles previos a ese evento. Los índices argentino y brasileño han superado notablemente el nivel que tenían antes de ese derrumbe.

Al considerar el inicio del periodo de análisis se puede ver también que el S\&P 500 crece sin verse afectado por el comportamiento de la bolsa de México durante la crisis económica que padeció éste. Los cuatro mercados reflejaron los efectos de la crisis asiática de 1997 y de la crisis de los bonos rusos en 1998. En tanto que para los tres países latinos se presentó un periodo de estancamiento en 1999, atribuible en parte a la crisis del mercado brasileño, al inicio del siglo actual la bolsa estadounidense observó una declinación en su nivel asociada en parte con el derrumbe de los valores tecnológicos después de la burbuja que se había dado en los precios de las acciones del sector asociado con el internet.

A partir de enero de 2003 se puede ver un alza interrumpida por el descenso ocasionado por la crisis de los valores asociados con hipotecas. Durante la caída de la bolsa de Estados Unidos en 2008 se observó también un descenso notorio en los índices de las tres bolsas latinoamericanas, aunque en el índice accionario argentino no parece ser tan grande, en realidad es por la escala de la figura.

Desde principios de 2020 el S\&P 500 inició un descenso sostenido hasta el mínimo ocurrido en marzo del mismo año, reflejo indiscutible no sólo de la incertidumbre provocada por la pandemia de Covid-19 sino también porque ésta se presentó en un entorno agravado por la guerra de precios petroleros desatada por Arabia Saudita en los primeros meses de año. Para mediados de agosto del mismo año ese indicador bursátil había vuelto a los niveles previos a la caída, prosiguiendo desde ese entonces la tendencia alcista que tenía antes del retroceso. Después de esa caída los índices bursátiles de los cuatro mercados han seguido un alza notoria, aunque en el caso del mercado argentino ha sido con mayor volatilidad. 

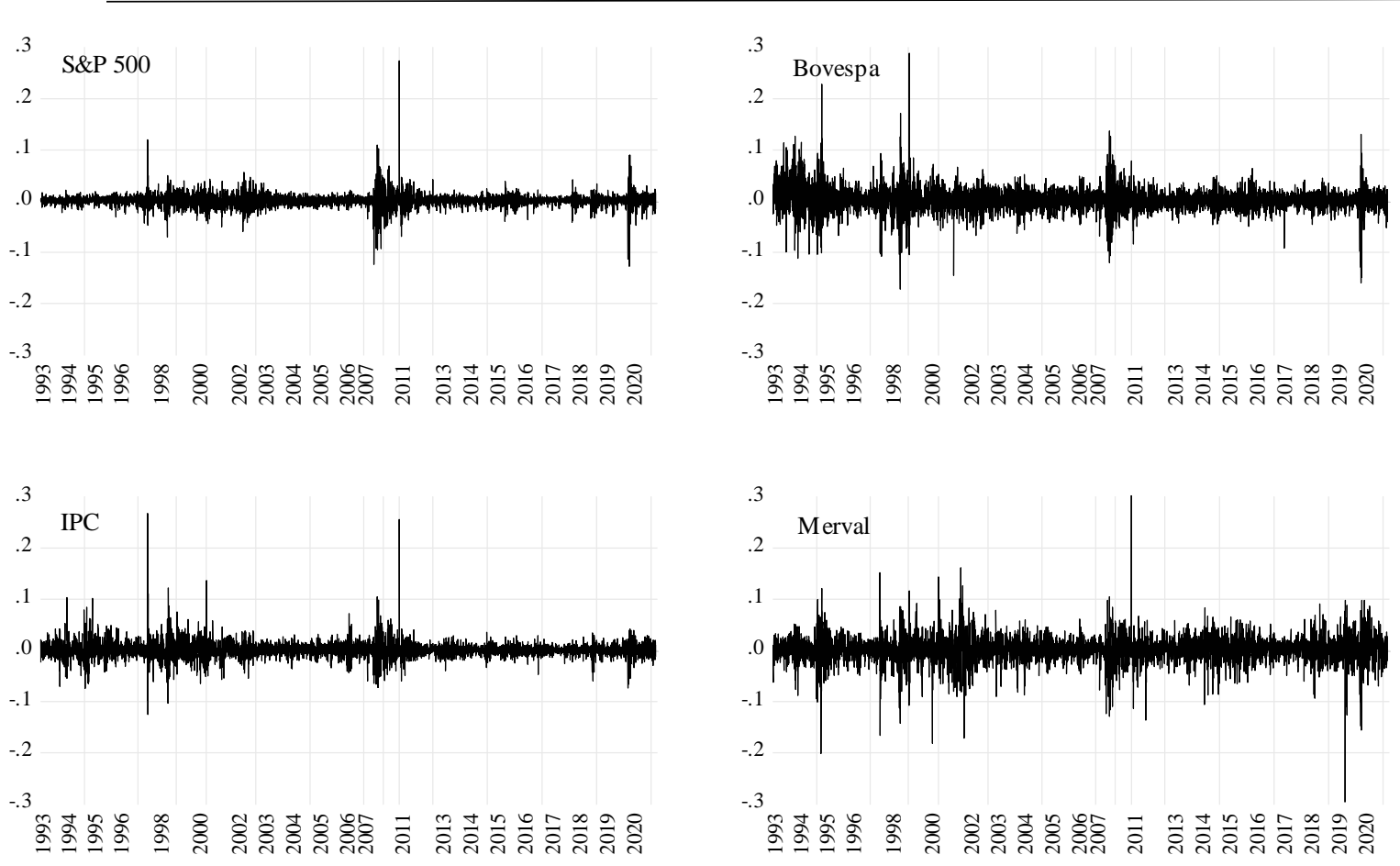

Figura 2. Rendimientos de los cuatro mercados bursátiles ${ }^{9}$

En la figura 2 se observa que la dispersión de los (log)rendimientos refleja los periodos de presión en las bolsas analizadas, haciendo que el comportamiento de los mercados latinoamericanos se asemeje, aunque con algunas particularidades. Los rendimientos de los mercados argentino y brasileño sugieren mayor volatilidad, lo que se corrobora con sus desviaciones estándar mostradas en el cuadro 1. A partir de la segunda década de este siglo, los rendimientos del mercado mexicano tienen una desviación estándar similar a la del mercado estadounidense que es la más baja. En el cuadro 2 se puede ver que las distribuciones de probabilidades de los rendimientos muestran sesgos positivos y elevada curtosis que llevan al rechazo de la hipótesis de normalidad según el estadístico de Jarque y Bera, notablemente con la mayor fuerza en los rendimientos de los mercados argentino y estadounidense.

Cuadro 1. Estadísticas básicas de los rendimientos

\begin{tabular}{|l|c|c|c|c|}
\hline & S\&P 500 & Bovespa & IPC & Merval \\
\hline Mínimo & -0.1277 & -0.1721 & -0.1253 & -0.4769 \\
\hline Cuartil 1 & -0.0041 & -0.0097 & -0.0065 & -0.0101 \\
\hline Mediana & 0.0005 & 0.0013 & 0.0004 & 0.0010 \\
\hline Media & 0.0004 & 0.0015 & 0.0006 & 0.0008 \\
\hline Cuartil 3 & 0.0056 & 0.0126 & 0.0077 & 0.0129 \\
\hline Máximo & 0.2740 & 0.2883 & 0.2675 & 0.6604 \\
\hline Desviación estándar & 0.0129 & 0.0234 & 0.0158 & 0.0269 \\
\hline
\end{tabular}

\footnotetext{
9 En la gráfica del mercado argentino (Merval) no se observan algunos rendimientos que superan el nivel de 0.3 esta, dado que son pocos se prefirió ocultarlos para no perder la perspectiva de comparabilidad con el resto de los mercados.
} 


\begin{tabular}{|l|c|c|c|c|}
\hline Sesgo & 1.2208 & 0.4024 & 1.7765 & 1.2355 \\
\hline Curtosis & 49.5903 & 11.0463 & 33.3408 & 85.7663 \\
\hline Jarque-Bera & $573,560.7$ & $28,540.9$ & $261,570.9$ & $1,712,883.1$ \\
\hline
\end{tabular}

En el cuadro 2 se muestran los resultados de las cópulas Clayton estimadas para considerar la estructura de dependencia en la cola inferior de las distribuciones bivariadas de las tres bolsas latinoamericanas y la bolsa de Estados Unidos. En los tres casos existen dos estados claramente diferenciados tanto por los parámetros $\theta$ de las cópulas estimadas como por los niveles de asociación medidos por la $\tau$ de Kendall. De acuerdo con la medida de dependencia en la cola, $\lambda_{I}$, el segundo estado queda identificado como el de un incremento en el grado de la dependencia y su probabilidad es mayor para los rendimientos del mercado mexicano ( $\approx 59 \%$ ) y notoriamente menor para los del brasileño ( $\approx 40 \%$ ), para los de Argentina es $\approx 52 \%$.

Es interesante observar que la fuerza de la asociación entre los rendimientos del Bovespa y el S\&P 500 casi se triplica cuando se encuentra en ese estado de dependencia más alta, en tanto que para la asociación de los rendimientos de los mercados mexicano y estadounidense aumenta a poco más del doble. Resalta el caso de los rendimientos del Merval, pues pasa de estar prácticamente sin asociación alguna en el estado uno a un alto grado equiparable al caso mexicano en el estado 2, alcanzando un enorme crecimiento que hace que sea poco más de 23 veces el nivel de dependencia observado en el estado de dependencia alta.

Cuadro 2. Cópulas con cambio markoviano entre dos regímenes

\begin{tabular}{|c|c|c|c|c|c|c|c|c|c|}
\hline \multicolumn{10}{|c|}{ Clayton } \\
\hline & \multicolumn{2}{|c|}{$p$} & \multicolumn{2}{|c|}{$\theta$} & \multicolumn{2}{|c|}{$\tau$} & \multicolumn{2}{|c|}{$\lambda_{I}$} & \multirow[b]{2}{*}{ CVM } \\
\hline Régimen & 1 & 2 & 1 & 2 & 1 & 2 & 1 & 2 & \\
\hline S\&P500-Bovespa & 0.6027 & 0.3973 & 0.4675 & 1.7490 & 0.1895 & 0.4665 & 0.2270 & 0.6728 & 0.2921 \\
\hline S\&P500-IPC & 0.4138 & 0.5862 & 0.5187 & 1.3176 & 0.2059 & 0.3972 & 0.2628 & 0.5909 & 0.4124 \\
\hline S\&P500-Merval & 0.4848 & 0.5152 & 0.1895 & 1.2474 & 0.0865 & 0.3841 & 0.0258 & 0.5737 & 0.2302 \\
\hline
\end{tabular}

En la figura 3 se muestran las probabilidades de encontrarse en el régimen identificado como el de mayor dependencia (régimen 2) en la cola inferior de la distribución bivariada de las tres bolsas latinoamericanas y la bolsa de Estados Unidos. Dichas probabilidades están condicionadas por el valor de la $\tau$ de Kendall de la observación del día de operación conjunta. En primera instancia se ven notorias diferencias en los patrones del movimiento conjunto, particularmente en el caso del mercado argentino en el que fluctúa de manera bastante aleatoria yendo de valores bajos a valores medianos la mayor parte del periodo, aunque en algunos lapsos se observan alzas a niveles cercanos del $80 \%$ de probabilidad. En el otro extremo, la bolsa mexicana muestra una cantidad mayor de dependencia alta incluso durante la mayor parte del periodo analizado, notablemente en los periodos asociados con crisis en los países emergentes a fines del siglo XX y desde inicios del presente siglo. El 
mercado brasileño muestra una mayor probabilidad de encontrarse en el estado de alta dependencia sólo durante los periodos críticos del presente siglo.
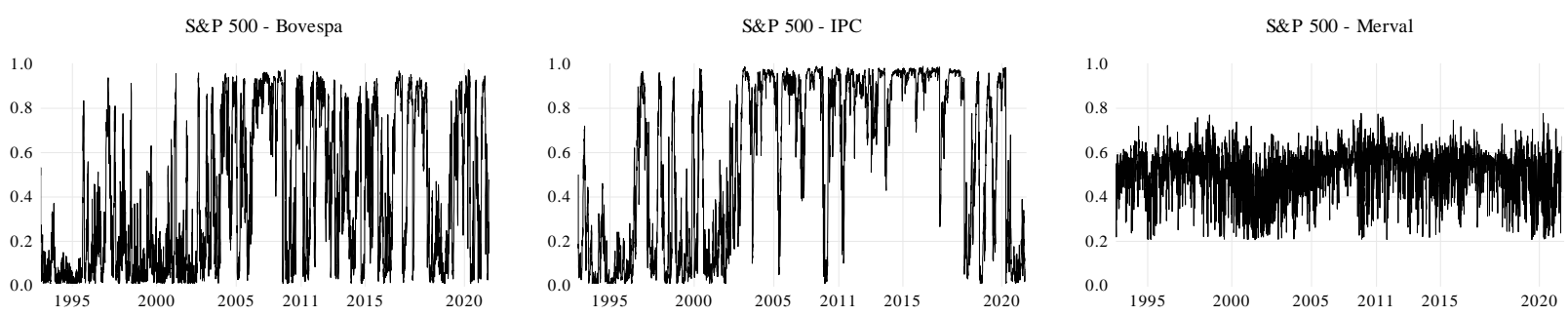

Figura 3. Probabilidades condicionales $\left(p_{2_{t}} \mid \tau_{t-1}\right)$ de la cópula Clayton

\section{Conclusiones}

Este estudio se suma a los análisis de los efectos críticos en los mercados financieros en periodos de turbulencia, en particular la suscitada como consecuencia de la irrupción de la pandemia de Covid19 y, de manera específica, para corroborar las diferencias y similitudes que existen entre esa y otras caídas de los mercados bursátiles de las tres principales economías de América Latina; tomando en consideración su asociación con el mercado estadounidense, es el más importante del mundo.

La crisis financiera observada en 2020 tuvo un origen distinto que las crisis previas, las cuales a fines del siglo pasado obedecieron a deficiencias en las estructuras económicas de los países emergentes, en tanto que las ocurridas durante el presente siglo han resultado de excesos especulativos en los mercados financieros. La pandemia provocó una crisis económica como ha sido ya analizado en una gran cantidad de artículos, sin embargo, se ha mostrado evidencia de que el comportamiento bursátil no es del todo distinto a los episodios previos. En el caso de los mercados accionarios de Brasil y México, la dependencia se ha vuelto más fuerte en la medida que ha aumentado la integración financiera de esos mercados y como consecuencia el grado de interacción dadas las conexiones establecidas en los circuitos financieros.

Aunque las dinámicas de los cuatro mercados analizados comparten similitudes claras, al menos durante una parte importante del horizonte temporal que cubre este estudio, es notorio que hay diferencias también muy claras. Entre esas diferencias destaca el menor crecimiento observado en las cotizaciones del mercado accionario mexicano a partir de 2017 y una menor fuerza en la tendencia de crecimiento posterior a la aparición de la pandemia causada por el Sars-Cov-2.

El comportamiento de los rendimientos de los mercados de capitales también refleja aspectos importantes. En particular, y como cabría esperar, se aprecia que los clústeres de volatilidad atienden a periodos similares, No obstante, la volatilidad de los mercados brasileño y argentino supera al del mercado bursátil de México, en especial en el periodo posterior a la declaración de la pandemia por Covid-19. Aunque no es el principal objetivo de este artículo, se requiere estudiar más los regímenes diferentes de volatilidad porque pueden tener implicaciones importantes para la cobertura de los riesgos de la inversión de portafolio.

Las diferencias en la forma en que se relacionan con el mercado estadounidense durante los periodos de crisis sugieren que en lo individual Argentina podría ser un refugio posible para crear una cobertura natural de los portafolios diversificados con activos estadunidenses y activos de ese 
país, en tanto que los mercados de Brasil y México podría no serlo. Sin embargo, también puede considerarse que tales diferencias muestran que en esos episodios una mayor diversión de los portafolios podría ser de utilidad si considera activos del mercado estadunidense y de los tres mercados latinoamericanos.

\section{Referencias}

[1] Abid, I., Kaabia, O. y Guesmi, K. (2014). Stock market integration and risk Premium: Empirical evidence for emerging economies of South Asia. Economic Modelling, 37(C), 408-416. https://doi.org/10.1016/j.econmod.2013.11.015

[2] Al-Awadhi, A. M., Alsaifi, K., Al-Awadhi, A., \& Alhammadi, S. (2020). Death and contagious infectious diseases: Impact of the COVID-19 virus on stock market returns. Journal of Behavioral and Experimental Finance, 27. https://doi.org/10.1016/j.jbef.2020.100326

[3] Ali, M., Alam, N., \& Rizvi, S. A. R. (2020). Coronavirus (COVID-19)—An epidemic or pandemic for financial markets. Journal of Behavioral and Experimental Finance, 27. https://doi.org/10.1016/j.jbef.2020.100341

[4] Akhtaruzzaman, M., Boubaker, S. y Sensoy, A. (2021). Financial contagion during COVID-19 crisis. Finance Research Letters, Volumen 38. https://doi.org/10.1016/i.frl.2020.101604

[5] Atri Hanen, Saoussen Kouki y Mohamed imen Gallali (2021). The impact of COVID-19 news, panic and media coverage on the oil and gold prices: An ARDL approach. Resources Policy, Volume 72, https://doi.org/10.1016/j.resourpol.2021.102061.

[6] Banco Interamericano de Desarrollo. (2014). MILA: Desafíos para la consolidación de un nuevo mercado bursátil más amplio e inclusivo RG-T2423. Banco Interamericano de Desarrollo: Washington

[7] Bartram, S., Taylor, S. y Wang, Y. (2007). The Euro and European financial market dependence. Journal of Banking \& Finance, 31,1461-1481. https://doi.org/10.1016/j.jbankfin.2006.07.014

[8] Bekaert, G. y Harvey, C. R. (2003). Emerging markets finance. Journal of Empirical Finance, 10(1-2), 355. https://doi.org/10.1016/S0927-5398(02)00054-3

[9] Bekaert, G., Harvey, C. y Ng, A. (2005). Market Integration and Contagion. The Journal of Business, 78(1), 39-70. http://dx.doi.org/10.1086/426519

[10] Boubaker, S. y Jouini, J. (2014). Linkages between emerging and developed equity markets: Empirical evidence in the PMG framework. The North American Journal of Economics and Finance, 29, 322-335. http://dx.doi.org/10.1016/j.najef.2014.06.004

[11] Bucio, C., Gutiérrez, R., y Sosa, M. (2017). Contagio vía cópulas dinámicas en los mercados de capitales del TLCAN de 2000 a 2016. EconoQuantum.Vol.16. Núm. 2, 1-23.v https://doi.org/10.18381/eq.v16i2.7072

[12] Cakmaklı, C., S. Demiralp, S, Kalemli-Ozcan, S. Yesiltas, y M. A. Yıldırım (2020). CoVID-19 and emerging markets: A SIR model, demand shocks and capital flows. Working Paper 27191, National Bureau of Economic Research.

[13] Calvo, S., y Reinhardt, C. (1996). Capital flows to Latin America: Is There Evidence of Contagion Effects? Policy Research Working Paper 1619.The World Bank, International Monetary Fund. http://dx.doi.org/10.1596/1813-9450-1619.

[14] Campos Marín, O. D., \& Revoredo Mendieta, L. A. (2017). Desafíos del mercado peruano en el MILA. The Latin American and Iberian Journal of Law and Economics, 3(2), 4; 94-110. 
[15] Carrieri, F., Errunza, V. y Hogan, K. (2007). Characterizing world market integration through time. Journal of Financial and Quantitative Analysis, 915-940. https://doi.org/10.1017/S0022109000003446

[16] Carvalho, L. (2018). Valsa brasileira: do boom ao caos econômico. Editora Todavia SA.

[17] Chambet, A., \& Gibson, R. (2008). Financial integration, economic instability and trade structure in emerging markets. Journal of International Money and Finance, 27(4), 654-675.

[18] Chernov, M., D. Creal.D., y Höedahl, P. (2020). Sovering credit and exchange rate risks: Evidence from ASIA- Pacific local currency bonds. NBER working paper series. 1-58. https://doi.org/10.3386/w27500

[19] Cound, J. (2021). Financial stability and nonbank financial institutions: Lessons from COVID-19 What worked and what needs to be addressed. Journal of Securities Operations and Custody, 13 (2),107-126. https://www.ingentaconnect.com/content/hsp/jsoc/2021/00000013/00000002/art00002

[20] de Souza, J.P. y Gómez-Ramírez, L. (2018). The paradox of Mexico's export boom without growth: a demand-side explanation. Structural Change and Economic Dynamics. 47, pp. 96-113.

[21] Damill, M., Frenkel, R., y Rapetti, M. (2015). Macroeconomic policy in Argentina during 2002-2013. Comparative Economic Studies, 57(3), pp. 369-400.

[22] Dima, B., Dima, Ş. M. y Barna, F. (2015). A wavelet analysis of capital markets' integration in Latin America. Applied Economics, 47(10), 1019-1036. http://doi.org/10.1080/00036846.2014.987917

[23] Dorodnykh, E. (2014). Determinants of stock exchange integration: evidence in worldwide perspective. Journal of EconomicStudies, 41(2), 292-316.

[24] Embrechts, P., Resnick, Sidney I. y Samorodnitsky, G. (1999) Extreme Value Theory as a Risk Management Tool, North American Actuarial Journal, 3 (2), 30-41, DOI: $\underline{10.1080 / 10920277.1999 .10595797}$

[25] Espinosa-Méndez, C., Gorigoitía, J., \&Vieito, J. (2017). Is the Virtual Integration of Financial Markets Beneficial in Emerging Markets? Evidence from MILA. Emerging Markets Finance and Trade, 53(10), 2279-2302.

[26] Forbes, K. J., y Rigobon, R. (2002). No Contagion, Only Interdependence: Measuring Stock Market Comovements? Journal of Finance, 57(5): 2223- 2261. http://dx.doi.org/10.1111/0022-1082.00494

[27] Frenkel, R. (2003). Globalización y crisis financieras en América Latina. Revista de la CEPAL, No. 80, 41-54.

[28] Fry-McKibbin, R., Hsiao, C. Y. L. y Martin, V. L. (2021). Measuring financial interdependence in asset markets with an application to eurozone equities. Journal of Banking \& Finance, 122(105985). https://doi.org/10.1016/j.jbankfin.2020.105985

[29] Ftiti, Zied; Hachmi Ben Ameur y Louhichic, Waël (2021). Does non-fundamental news related to COVID-19 matter for stock returns? Evidence from Shanghai stock market. Economic Modelling, Volumen 99, https://doi.org/10.1016/j.econmod.2021.03.003

[30] García, D. A., \& Rosso, J. (2016). Efectos estacionales en los mercados de capitales de la Alianza del Pacífico. Estudios Gerenciales, 32(141), 358-368.

[31] Gil, U., Jorge, M., \& Mosquera López, S. (2014). Efectos del MILA en la eficiencia de portafolio de los mercados de acciones colombiano, peruano y chileno. Cuadernos de Administración (Universidad del Valle), 30(52), 75-83.

[32] Gjika, D. y Horvath, R. (2013). Stock market comovements in Central Europe: Evidence from the asymmetric DCC model. Economic Modelling, 33, 55-64. http://dx.doi.org/10.1016/j.econmod.2013.03.015

[33] Grabel, J. (2021). Market corrections, panics, and Covid-19. Journal of Financial Service Professionals, 75 (1), 10-13 
REMEF (The Mexican Journal of Economics and Finance)

Dependencia de los mercados accionarios de Argentina, Brasil y México respecto del

estadounidense: Covid19 y otras crisis financieras recientes

[34] Hernández, L. F., y Valdés, R. O. (2001). What drives contagion: trade, neighborhood, or financial links? International Review of Financial Analysis, 10(3), 203-218

[35] Karpoff, J. M. (1987). The relation between price changes and trading volume: A survey. Journal of Financial and quantitative Analysis, 22(1), 109-126.

[36] Kasa, K. (1992). Common stochastic trends in international stock markets. Journal of Monetary Economics, 29(1), 95-124. https://doi.org/10.1016/0304-3932(92)90025-W

[37] Kenourgios, D., Samitas, A. y Paltalidis, N. (2011). Financial crises and stock market contagion in a multivariate time- varying asymmetric framework. Jornal of International Financial Markets, Institutions \& Money, 21(1), 92-106. https://doi.org/10.1016/j.intfin.2010.08.005

[38] Kumamoto, M., y Zhuo, J. (2020). Government Bond Market Integration in ASEAN Countries. Asian $\begin{array}{llll}\text { Economic and } & \text { Financial }\end{array}$ https://doi.org/10.18488/journal.aefr.2020.103.289.312

[39] López, H, F., Ortiz, E. y Cabello, A. (2009). Las interrelaciones de volatilidad y rendimientos entre los mercados de valores del TLCAN. Investigación económica, 68(267), 83-114. http://dx.doi.org/10.22201/fe.01851667p.2009.267.37389

[40] López-Herrera, F., \& Ortiz, E. (2010). Cointegration trends among the NAFTA equity markets. Revista de Economía Mundial, (26), 155-176.

[41] López, H, F. y Venegas, M. F. (2012). Integración Financiera México-Estados Unidos: mercados accionarios y de derivados accionarios. Economía: teoría y práctica, (36), 179-196.

[42] López, H, F., Benavides, D., \& Gurrola, Ríos C. (2019). Spillovers entre el S\&Poor500 y los principales EMBIG latinoamericanos. Revista Mexicana de Economía y Finanzas Nueva Época REMEF, 14, 527-540.

[43] Löwen C, Kchouri B. y Lehnert T. (2021) Is this time really different? Flight-to-safety and the COVID19 crisis. PLoS ONE 16(5). https://doi.org/10.1371/journal.pone.0251752

[44] Marcet, F. (2017). Analyst coverage network and stock return comovement in emerging markets. Emerging Markets Review, 32, 1-27.

[45] Martínez, D., Narváez, G. y Echeverry, M. (2012). Ventajas del Mercado Integrado Latinoamericano MILA. Disponible en: http://hdl.handle.net/10818/3943; consultado el 8 de octubre de 2018.

[46] Mellado, C., \& Escobari, D. (2015). Virtual integration of financial markets: a dynamic correlation analysis of the creation of the Latin American Integrated Market. Applied Economics, 47(19), 19561971.

[47] Mendoza, V., A., López, H., F. y Watkins, F., K. (2011), Reflexiones sobre las crisis financieras, Centro de Investigación e Inteligencia Económica CIIEUPAEP y División de Investigación de la Facultad de Contaduría y Administración, UNAM. Coordinadores.

[48] Mollah, S., y Hartman, T. (2012). Stock Market Contagion, Interdependence and Shifts in Relationship due to Financial Crisis-A Survey. International Review of Business Research Papers, 8(1), 166-195.

[49] Mohti, W., Dionísio, A., Vieira, I. y Ferreira, P. (2019) Financial contagion analysis in frontier markets: Evidence from the US subprime and the Eurozone debt crises. Physica A: Statistical Mechanics and its Applications, 525,1388-1398. https://doi.org/10.1016/j.physa.2019.03.094

[50] Moreno-Brid, J. C., y Ros, J. (2009). Development and growth in the Mexican economy: a historical perspective. Oxford - New York: Oxford University Press.

[51] Moreno-Brid, J. C., \& Ros, J. (2010). La dimensión internacional de la economía mexicana. In S. Kuntz Fischer (Ed.), História económica general de México. De la Colonia a nuestros días (pp. 757-788). México: El Colegio de México - Secretaría de Economía. Págs. 757-788. 
[52] Munteanu, A., Filip, A. y Pece, A. (2014). Stock market globalization: the case of Emerging European Countries and the US. Procedia Economics and Finance, 15, 91-99. http://dx.doi.org/10.1016/S22125671(14)00452-3

[53] Narayan, S., Sriananthakumar, S. y Islam, S. Z. (2014). Stock market integration of emerging Asian economies: Patterns and causes. Economic Modelling, 39, 19-31. https://doi.org/10.1016/j.econmod.2014.02.012

[54] Nelsen, R.B. (2006). An Introduction to Copulas, $2^{\text {nd }}$ ed. Springer, New York.

[55] Nguyen, C., Bhatti, M. I. y Henry, D. (2017). Are Vietnam and Chinese stock markets out of the US contagion effect in extreme events? Physica A: Statistical Mechanics and its Applications, 480, 10-21. http://dx.doi.org/10.1016/j.physa.2017.02.045

[56] Palazzo, G., y Rapetti, M. (2017). Real exchange rate and export performance in Argentina, 2002-2008. Journal of post Keynesian economics, 40(1), pp. 75-94.

[57] Rigobon, R (2002). International Financial Contagion: Theory and Evidence in Evolution, CFA. The Research Foundation Publications.

[58] Rojas, J. A. O., \& Castro, F. A. T. (2016). El Mercado Integrado Latinoamericano (MILA): sus expectativas y el impacto del latibex. Sinapsis, 8(2), 121-145.

[59] Romero-Álvarez, Y. P., Ramírez-Atehortúa, F. H. y Guzmán-Aguilar, D. S. (2013). Mercado Integrado Latinoamericano (MILA): análisis de correlación y diversificación de los portafolios de los tres países miembros en el período 2007-2012. Cuadernos de Contabilidad, 34(14), 53-74.

[60] Shahateet, M. (2019). Testing the linkages of Arab stock markets: a multivariate GARCH approach. Investment Management \& Financial Innovations, 16(4), 192-204. http://dx.doi.org/10.21511/imfi.16(4).2019.17

[61] Sandoval-Alamos, E., Vásquez-Párraga, A. Z., \& Sabat-Arriagada, R. (2015). Integration of the stock markets of Chile, Colombia and Peru in The Latin American Integrated Market (MILA). Innovar: Revista de ciencias administrativas y sociales, 25(Spe), 71-84.

[62] Sandoval, E., \& Soto, M. (2016). Mercado integrado latinoamericano: un análisis de cointegración. Revista Internacional Administración \& Finanzas, 9(2), 1-17.

[63] Santillán, S, R. J. (2015). La Gran Recesión (2007-2012): Lecciones y Oportunidades para México. EGADE Business School, Instituto Mexicano de Ejecutivos de Finanzas, PWC.

[64] Santillán, S, R. J., Gurrola, R., C. y López, H., F. (2017). Evaluación del grado de integración de los principales mercados de capital europeos con un modelo Cópula-GARCH. Estocástica: finanzas y riesgo, 6(1), 9-36.

[65] Schweizer, B. y Wolff, E. F. (1981). On Nonparametric Measures of Dependence for Random Variables. The Annals of Statistics, 9(4), 879-885.

[66] Sklar, A. (1959) Fonctions de Répartition à n Dimensions et Leurs Marges. Publications de l'Institut Statistique de l'Université de Paris, 8, 229-231.

[67] Sosa, M., Ortiz, E., \& Cabello, A. (2018). Dynamic Linkages between Stock Market and Exchange Rate in MILA Countries: A Markov Regime Switching Approach (2003-2016). Revista Análisis Económico, 33(83), 57-74.

[68] Trabelsi, M. A., y Hmida, S. (2018). A dynamic correlation analysis of financial contagion: Evidence from the Eurozone stock markets. Entrepreneurial Business and Economics Review, 6(3), 129-141. https://doi.org/10.15678/EBER.2018.060308

[69] Valadkhani, A. y Chancharat, S. (2008), Dynamic linkages between Thai and international stock markets, Journal of Economic Studies, 35(5),425-441. https://doi.org/10.1108/01443580810903572

[70] Vargas, P. W., \& Bayardo, M. J. (2013). El MILA. Mercado de integración entre Chile, Perú y Colombia. Revista Relaciones Internacionales, Estrategia y Seguridad, 8(1), 113-133. 
REMEF (The Mexican Journal of Economics and Finance)

Dependencia de los mercados accionarios de Argentina, Brasil y México respecto del estadounidense: Covid19 y otras crisis financieras recientes

[71] Veerappa, B. S. (2016). Cointegration of Asian Stock Markets: Empirical Evidence from India. International Journal of Financial Management, 6(2), 25-40. http://dx.doi.org/10.21863/IJFM/2016.6.2.030

[72] Worthington, A. C., \& Higgs, H. (2006). Evaluating financial development in emerging capital markets with efficiency benchmarks. Journal of Economic Development, Vol. 31, issue 1, 17-44. 